\title{
Utilizing microblogs for optimized real-time resource allocation in post-disaster scenarios
}

\author{
Moumita Basu' ${ }^{1} \cdot$ Sipra Das Bit ${ }^{2} \cdot$ Saptarshi Ghosh $^{3}$
}

Received: 4 June 2021 / Revised: 30 August 2021 / Accepted: 8 November 2021 / Published online: 8 December 2021

(c) The Author(s), under exclusive licence to Springer-Verlag GmbH Austria, part of Springer Nature 2021

\begin{abstract}
In the aftermath of a disaster event, it is of utmost important to ensure efficient allocation of emergency resources (e.g. food, water, shelter, medicines) to locations where the resources are needed (need-locations). There are several challenges in this goal, including the identification of resource-needs and resource-availabilities in real time, and deciding a policy for allocating the available resources from where they are available (availability-locations) to the need-locations. In recent years, social media, and especially microblogging sites such as Twitter, have emerged as important sources of real-time information on disasters. There have been some attempts to identify resource-needs and resource-availabilities from microblogging sites. However, there has not been much work on having a policy for optimized and real-time resource allocation based on the information obtained from microblogs. Specifically, the allocation of critical resources must be done in an optimal way by understanding the utility of emergency resources at various need-locations at a given point of time. This paper attempts to develop such a utility-driven model for optimized resource allocation in a post-disaster scenario, based on information extracted from microblogs in real time. Experiments show that the proposed model achieves much better allocation of resources than baseline models-the allocation by the proposed model is not only more efficient in terms of quickly bringing down resource-deficits at various need-locations, but also more fair in distributing the available resources among the various need-locations.
\end{abstract}

Keywords Disaster $\cdot$ Post-disaster relief $\cdot$ Resource-need $\cdot$ Resource-availability $\cdot$ Optimization $\cdot$ Resource utility

\section{Introduction}

In recent years, several major natural disasters such as hurricanes, earthquakes, and floods have caused massive devastation and loss of life and assets around the world ( $\mathrm{Li}$ et al. 2017; Radianti et al. 2015). In the aftermath of any disaster, a crucial step is to coordinate the distribution of emergency resources from different warehouses (where resources are available) to various need-locations (where resources are needed) in real time. However, according to the World Disaster Report 2018, ${ }^{1}$ millions of victims in crisis do

Moumita Basu

moumitabasu0979@gmail.com

1 Present Address: Amity University Kolkata, Kolkata 700156, India

2 Indian Institute of Engineering Science and Technology Shibpur, Howrah 711103, India

3 Indian Institute of Technology Kharagpur, Kharagpur 721302, India not receive the vital resources they urgently need, primarily due to two reasons-(i) lack of first-hand information about resource-needs and resource-availabilities in real time, and (ii) lack of appropriate resource allocation strategies in post-disaster scenario when the availability of emergency resources is often scarce and constrained.

Evidently, the first challenge is to decide how to obtain information of resource-needs and resource-availabilities in real time. Many recent studies have shown that Online Social Media, especially microblogging sites such as Twitter and Weibo, are important repositories of real-time information during disasters (Imran et al. 2015; Varga et al. 2013; Rudra et al. 2015; Bhavaraju et al. 2019; Pourebrahim et al. 2019; Hiltz et al. 2020). There are three challenges in utilizing microblogs for post-disaster resource allocation-(i) identifying tweets informing about needs of resources (which we call as a need-tweet) and availability of resources (which we call as an availability-tweet), from among thousands of conversational/opinion tweets, (ii) extracting essential

\footnotetext{
$\overline{1}$ https://media.ifrc.org/ifrc/world-disaster-report-2018/.
} 
information like resource name, resource quantity and location names from the need-tweets and availability-tweets, and (iii) matching resource-needs and resource-availabilities in such a way that allocation of scarce resources is optimal.

In this scenario, our broad objective is to develop a holistic approach for efficient distribution of emergency resources in a post-disaster situation, utilizing real-time information available on social media (specifically Twitter). To this end, our prior works have already proposed methodologies for the first challenge stated above, i.e. for identification of needtweets and availability-tweets (Basu et al. 2019, 2017a). We have also developed a mechanism for understanding needtweets and availability-tweets, and for extracting critical information such as name and quantity of resource, location name from such tweets (i.e. the second challenge) in another prior work (Dutt et al. 2019). However, there has not been much work on the third challenge, namely optimal allocation of resources based on needs and availabilities extracted in real time from social media.

It can be noted that, in our prior work (Dutt et al. 2019), we developed an algorithm for matching need-tweets with availability-tweets considering overlapping mentions of resources and geographical proximity between the needlocation and the availability-location. However, this methodology considered each need-tweet and each availabilitytweet in isolation (i.e. individually). For instance, the same resource-availability of 100 units of resource R 1 can be chosen to match two resource-needs for R1, both of which need 80 units of R1—evidently, this matching cannot happen in practice. Hence, the methodology of (Dutt et al. 2019) is not suitable in practical scenarios, where there are many resource-needs and resource-availabilities appearing together. Hence, in the present work, we have developed a framework to match a sequence of resource-needs and a sequence of resource-availabilities, considering a utilitydriven model for optimal allocation of resources.

Our framework is based on a prior work by Basu et al. (2018b) who formulated a resource allocation strategy considering the demand and utility of resources. However, Basu et al. considered data extracted from Government reports that are compiled months after the actual disaster event occurred (details given in Sect. 2). These reports are complete and accurate but not real time. Thus, Basu et al. considered a fixed set of shelter-points as the need-locations (where resources are needed), and a fixed set of warehouses as the availability-locations (where resources are available). Also, Basu et al. assumed complete knowledge of resource needs and allocations at every shelter-point on every day. In practice, in a post-disaster scenario, need-locations and availability-locations can change on a day-to-day basis for various reasons, such as aftershocks of an earthquake, or flash floods affecting new regions. Also, information about resource-needs and resource-availabilities is very sparse on social media, and it is not practical to assume complete knowledge of needs and allocations on a day-to-day basis. Thus, the proposed scheme of Basu et al. (2018b) is not flexible enough to deal with the dynamic scenario in a post-disaster situation, as is observed from microblog streams collected in real time.

In the present work, we have adapted the model by Basu et al. (2018b) to make it suitable for real-time resource allocation in a practical post-disaster situation. First, we propose to employ a semi-automatic approach based on the methodology developed in our prior work (Dutt et al. 2019) to extract need-tuples from need-tweets and availability-tuples from availability-tweets. These need-tuples and availability-tuples store critical information such as the name of the resource needed/available, the quantity that is needed/ available, the location where the resource is needed/available, etc. The resource allocation model takes as input these tuples extracted from the microblogs posted every day. Next, we propose several changes to the existing model of Basu et al. (2018b), that include-(i) considering variations in the set of need-locations and the set of availability-locations with time (which can change in practice in a disaster scenario), (ii) making the utility computation tolerant to missing information, such as computing utility of a resource at a need-location for which there is no prior information on the amount of resources allocated earlier (which was not possible in the original model), (iii) considering unfulfilled resource-needs and residual resource-availabilities on a certain day $t$ while doing the resource allocation on the next day $t+1$, and so on. All these adaptations make the model developed in this work much more suitable for application on information collected from social media sites such as Twitter (which is real time but can be incomplete and sparsely available).

Using a working example derived from tweets posted during a real-life disaster event, we show that our proposed model results in more efficient resource allocation than some baseline approaches including that of our prior work (Dutt et al. 2019). Specifically, the proposed model can bring down the resource deficit at various need-locations much more quickly, compared to the baseline approaches. We also show that our proposed model leads to resource allocation that is more fair - other approaches may result in a situation where resources are allocated to only a subset of need-locations, while others face starvation; in contrast, our proposed model attempts to distribute the resources more evenly, so that no need-location faces starvation of resources. We believe that our proposed model would help disaster management authorities in allocating resources more efficiently and effectively in real time, during future disaster events.

Thus, in the present work, our main contributions can be summarized as follows: 
- To the best of our knowledge, we make the first attempt to develop a resource allocation framework utilizing social media data for optimized, real-time resource allocation in post-disaster scenarios. Different from prior post-disaster resource allocation models (such as (Basu et al. 2018b)), our framework handles unfulfilled needs and surplus availabilities in need-locations and availability-locations, respectively, and can also handle new locations and resources evolving dynamically.

- We show that our proposed framework can bring down resource-deficits in need-locations more efficiently than prior models using a small working example derived from need-tweets and availability-tweets posted during a real-world disaster event-severe floods in the Indian city of Chennai in 2015. Specifically, Average Resource Deficit (ARD), which measures the rate at which overall resource deficit is minimized across all need-locations, is seen to be reduced by a much larger extent (between $20 \%$ and $40 \%$ ) by our method, as compared to methods that consider each need and availability separately.

- We also show that the resource allocation by our model is likely to be more fair (i.e. less chances of a particular need-location facing starvation for resources) than the allocation by methods that consider each need and availability separately. This fairness in allocation usually comes at the cost of slightly increased deployment time of resources.

The rest of the paper is structured as follows. Section 2 describes related work relevant to the present work. Section 3 describes a methodology for extracting need-tuples and availability-tuples from microblogs. Section 4 describes the resource allocation models; specifically, Sect. 4.2 details the model by Basu et al. (2018b), while Sect. 4.3 describes our proposed modifications to the model. Section 5 describes experiments using a working example, where the proposed model is compared with some baseline approaches for resource allocation. Finally, the paper is concluded in Sect. 6 .

\section{Related work}

In the past few decades, OSM has become an important source of first-hand information during disasters such as earthquakes, floods, hurricanes, terror attacks, and so on. It has been observed that during mass emergencies, victims and other responding organizations are utilizing Facebook and Twitter as an alternative communication mechanism to reach out to emergency services. Hence, several academicians have advocated the importance of online social media in disaster resilience (Yadav and Rahman 2016). Predominantly, microblogging sites like Twitter, Weibo have become a vital source of such critical information to assist post-disaster relief operations (Pohl et al. 2015; Xu et al. 2017). However, it has been observed that critical information is often obscured in a deluge of conversational posts (such as prayers for victim, sympathy, personal opinions). Thus, automatic extraction of informative tweets that provide useful information become challenging (Madichetty and Sridevi 2020a; Barnwal et al. 2019; Singh et al. 2019). Hence, in the past few years, there has been a lot of efforts towards extracting such critical information from OSM (predominantly Twitter) for helping in post-disaster relief operation. For instance, some prior works have focused on identifying location references from tweets during emergencies (Singh et al. 2019; Kumar and Singh 2019). Other prior works have also developed methodologies for utilizing multimodal Twitter data, e.g. for earthquake detection (Alqhtani et al. 2018). Kumar et al. (2020) developed a deep multi-modal neural network framework to identify disaster-related informative content from Twitter streams using text and images together. The readers are referred to (Imran et al. 2015; Li et al. 2017) for comprehensive surveys on efforts to utilize social media content during emergencies/disasters.

In the rest of this section, we focus on prior works specifically related to need and availability of resources in a post-disaster scenario.

\subsection{Identification of resource-needs and resource-availabilities}

Some prior studies focused on identifying information regarding need and availability of resources in a disaster scenario. Tweets providing such information are usually referred to a 'need-tweets' and 'availability-tweets'. To this end, (Varga et al. 2013) used NLP-based methodologies on Japanese tweets posted during an earthquake in Japan. (Purohit et al. 2014) developed a set of 18 regular expressions to extract specific types of need-tweets indicating donation requests for different resources, and tweets representing the availability of resources to be donated. A recent study (Madichetty and Sridevi 2020b) utilized a majority voting-based ensemble method to specifically identify tweets related to the requirement and availability of medical resources during a disaster, in order to aid medical organizations and victims. In another recent study, (Purohit et al. 2020) developed a serviceability model and a system to identify and rank highly serviceable requests during a disaster. The system also possesses a facility to re-rank service requests by semantic grouping to reduce redundancy and to assist the browsing of requests.

In our prior works (Basu et al. 2019, 2017a; Khosla et al. 2017), we employed several supervised and unsupervised methodologies to address the problem. In the supervised approach, we experimented with several traditional and 
neural network-based classifiers trained on microblogs posted during prior disaster events, that can be applied to microblogs posted during an unseen disaster event for identifying need-tweets and availability-tweets. We also used unsupervised pattern matching and information retrieval methodologies that can be directly employed on the microblogs posted during an ongoing disaster event.

\subsection{Matching resource-needs and resource-availabilities}

There have also been a few attempts to address the problem of matching need-tweets and availability-tweets. Purohit et al. (2014) developed methodologies to match need-tweets and availability-tweets by matching regular expressions and using TF-IDF vectors as features. (Bhoi et al. 2020) developed a deep learning-based hybrid model utilizing word-embeddings, CNN, and LSTM to identify multilingual need-tweets and availability tweets. Subsequently, they proposed a methodology to map need-tweets with matching availability tweets by utilizing a two-word sliding window approach used to generate the combined embedding of two adjacent words. In our prior works (Dutt et al. 2019; Basu et al. 2018a), we developed methodologies to match needtweets and availability-tweets considering both resource similarity and geographical proximity between locations of need and availability.

However, in all of these prior works, each need-tweet and availability-tweet is treated individually. Thus, one needtweet can be matched to multiple availability tweets. In reality, there will be many demands for the same resource (e.g. at different locations), and hence, the available resources need to be allocated intelligently to all those demands - this situation is not handled by the studies mentioned above. Importantly, prior matching algorithms do not ensure the allocation of resources in an optimal way.

\subsection{Optimizing resource allocation}

Optimization techniques have been employed to solve a broad category of problems, ranging from rumor detection from twitter data using optimized shallow classifiers (Kumar et al. 2019) to speech emotion recognition using discriminative dimension reduction technique (Daneshfar and Kabudian 2020). But to our knowledge, there are only two prior works that attempted to optimize resource allocation specifically for a post-disaster scenario (Basu et al. 2018b; Schempp et al. 2019).

Basu et al. (2018b) proposed a model for utility-based resource allocation for post-disaster relief operations. Specifically, their model was based on data about a multi-day cloudburst in 2013 in the Uttarakhand state of India. They collected data of resource-needs and resource-availabilities at various shelter points/warehouses from the web-portal of the Uttarakhand Government and developed a resource allocation model based on these data. Note that, the data were collected in an offline manner, several months after the disaster occurred. As a result, the situation considered in this study is relatively static - the set of shelter points and warehouses remain fixed throughout, and demands/ availabilities of resources at these shelter points/warehouses are assumed to be known regularly at the end of each day. Thus, the framework in (Basu et al. 2018b) is difficult to apply for real-time resource allocation in the immediate aftermath of a disaster, where the need-locations and availability-locations can vary from day to day, and the allocation has to be carried out based on information obtained from social media (which is the most common source of real-time information today). Utilizing the sporadic OSM data for resource allocation in real time during a disaster has several challenges, as will be illustrated in the next section.

These limitations were partially addressed in the more recent work by Schempp et al. (2019), who proposed a model to optimize disaster relief distribution using Global Particle Swarm Optimization and Mixed-Integer Linear Programming. This study (Schempp et al. 2019) considered both social media data and authoritative data (collected from ground surveys, cadastral surveys, census, etc.) to model the rescue demand requests. The model used rescue demand requests and location of demand from social media data as demand points. Also, additional simulated demand points were generated from the authoritative data. On the other hand, locations of the hospitals were extracted from authoritative data and were used as the connector to simulated temporal stations or rescue centres perceived as distribution centres for first-aid and supplies in disaster situations. Thus, the framework in Schempp et al. (2019) does not support fully real-time allocation of resources, since resource-availability data are not extracted from real-time social media data. Another limitation of the framework in Schempp et al. (2019) is that no concept of utility/priority of various resources is considered (unlike in Basu et al. (2018b)).

Since the objective of the present study is resource allocation in a real-time setting, based on data collected dynamically from OSM, we adapt the utility model and resource deployment model of Basu et al. (2018b) with significant modifications (that are detailed in later sections). Different from (Schempp et al. 2019), our framework considers both resource-needs and resource-availabilities to be extracted in real time from social media and also considers utility/priority of resources. The introductory concept of this study was introduced in our prior work (Basu et al. 2021). However, our prior work only introduced the idea of the framework proposed in the present manuscript and did not contain any of the detailed analyses reported in the manuscript. 
Table 1 Some examples of resources of the five classes, that are commonly required in a post-disaster scenario

\begin{tabular}{ll}
\hline Category & Examples \\
\hline Health & Blood, anaesthetic, antibiotics, latrines, tissue paper, sanitary napkins, soap bars \\
Shelter & Tents, rope, tarpaulins, sheets, blankets, clothes, shelter kit, jackets, boots, gloves, camp \\
Food & Cereal, bottled water, canned food, utensils, fuel, dried fruits, biscuits, vegeTables, \\
Logistics & Electricity, storage, doctors, army, power, helicopters, communication, volunteers, \\
Cash & Funds, money, supplies, donations, stock
\end{tabular}

\section{Identifying need-tuples and availability-tuples from microblogs}

As stated in Sect. 2, several methodologies have been developed for identifying 'need-tweets' and 'availability-tweets' that inform of resource-needs and resource-availabilities, respectively (Basu et al. 2017a, 2019; Khosla et al. 2017). We assume that such methods are first applied to identify need-tweets and availability-tweets from tweet streams. Subsequently, the specific resource-needs are extracted from need-tweets in the form of need-tuples each of which is of the form \{Resource, Location, Quantity, Emergency score (each field is explained below). Similarly, the resourceavailabilities are extracted from availability-tweets in the form of availability-tuples, each of which is of the form \{Resource, Location, Quantity\}. In this section, we describe the methodology to extract the tuples from need-tweets and availability-tweets.

\subsection{Parsing need-tweets and availability-tweets}

In our prior work (Dutt et al. 2019), we developed a parser that operates on the text of need-tweets and availabilitytweets to extract five useful information-resource name, quantity, location, source, and contact. We briefly describe how the parser works.

Potential resource names are extracted by obtaining a dependency tree by employing dependency parsing on preprocessed tweet text. After extracting potential resources from the text of a tweet, semantic similarity of the extracted words is verified with an exhaustive list of resources that are predominantly required in disaster situations. The aforementioned list of resources is compiled following the guidelines of the United Nations Office for the Coordination of Humanitarian Affairs (UNOCHA). ${ }^{2}$ As per the classification of UNOCHA, five broad classes of resources are commonly required in the aftermath of a disaster, namely Food, Shelter, Logistic, Health, and Cash. Table 1 shows examples of each class of resources. Such resource names are extracted by the parser developed in (Dutt et al. 2019). Likewise, numeric tokens such as a real number (say '500')

\footnotetext{
$\overline{2}$ https://vosocc.unocha.org/getFile.aspx?file=att36103_h4t800.pdf.
}

or a semantic representation of a real number (say 'ton') are identified as quantity if in the tweet text it is followed by at least one resource name. For identifying geographical locations, a combination of various Natural Language Processing techniques are used, that include hashtag segmentation, POS (Parts-of-Speech) tagging, Regex matches, and dependency parsing of need-tweets and availability-tweets considering dependency distance (Dutt et al. 2019). Named Entity Recognition (NER) taggers are also used to identify named entities that conform to locations (specifically, entities for which GPE, LOC and FACILITY tags are reported by the NER tagger). Finally, the extracted potential locations are verified using Open Street Map gazetteer (details in (Dutt et al. 2019)). The gazetteer also returns the geo-spatial coordinates of the locations, which have several utilities. For instance, the geo-spatial coordinates enable us to calculate the distance between the locations where a resource is needed and where it is available. Again, the geo-spatial coordinates can help in location name disambiguation, e.g. by checking whether the coordinates are within a 'bounding box' around the region affected by the present disaster.

In our prior work (Dutt et al. 2019), we applied the dependency parser (described above) on tweets collected during three different disaster events, namely an earthquake in Nepal (for which we had 499 need-tweets and 1, 333 availability-tweets), an earthquake in Italy (having 177 need-tweets and 233 availability-tweets) and floods in the Indian city of Chennai (having 4, 516 need-tweets and 6, 583 availability-tweets). We evaluated the performance of the parser using gold standard data sets annotated by human annotators. We observed that the parser performed reasonably well in extracting the resource, location, and quantity for all the disaster events. The $F$-Score value for extracting resource and location was higher than 0.8 and was 0.78 for the quantity extraction. Further details of the evaluation process can be found in our prior work (Dutt et al. 2019).

In the present study, we applied our parser to extract need-tuples and availability-tuples from the need-tweets and availability-tweets, respectively, where each tuple consisting of three fields-(i) resource name, (ii) quantity that is needed/available, and (iii) location where the resource is needed/available. Additionally, a fourth field is included in a need-tuple-an emergency score. The emergency score is assigned to each need-tuple corresponding to the urgency/ 
priority of the corresponding need. For instance, the needs of senior citizens, babies, and pregnant women can be given higher priority while allocating resources. In this work, for simplicity, we have considered only two levels of the emergency score, i.e. Norm_Emergency_Score $=10$ (normal priority) and Max_Emergency_Score $=20$ (higher urgency or priority). However, the emergency score can be decided by the responding authorities as appropriate.

\subsection{Challenges in extracting need-tuples and availability-tuples}

As stated above, we used an automatic parser (developed in our prior work (Dutt et al. 2019)) to identify resource names, quantity and location from the need-tweets and availabilitytweets. However, we also observed several challenges in such automatic extraction of need-tuples and availabilitytuples. We now describe these challenges; we also demonstrate the challenges by giving some examples of tweets from which automatic extraction of critical information (resource, quantity, location, etc.) is challenging. The examples are taken from tweets posted during the severe floods in the Indian city of Chennai, in December 2015 (details of this data set can be found in our prior work (Dutt et al. 2019)).

Tweets are often written in an informal way and sometimes critical information such as resource, location, quantity is mentioned indirectly. Moreover, people use diverse vocabulary for the same resource. The use of regional languages is prevalent; for instance, food is referred to as 'Langar', 'Chappati', 'Idly', etc., depending on the region where the disaster has occurred. Also, there is lot of ambiguity in microblogs. For instance, consider the following two tweets-(i) "INS Airawat equipped with 5 Jamini boats, 20 divers, lot to relief material, will reach Chennai today late eve or tomorrow morning", and (ii) "\#ChennaiFloods 11 boats incl Landing Craft +20 divers, Disaster \& Medical relief bricks on Airavat rushed to Chennai to augment Relief'. It is difficult to understand whether both tweets refer to the same resource-availabilities.

Moreover, the units used to specify the quantity of resources needed/available are also diverse. To deal with such wide variations in the style of writing microblogs, several assumptions have to be made, e.g. to unify the units of resource needs/availabilities. Some examples are given as follows:

- The quantity is mentioned in diverse units. For example, in the tweet "Two families struck with a 3month baby Kindly help No 7, kalaivanar strt, parvathy Nagar. mudichur road Chennai", the unit stated is 'family'. However, we are considering the person count as a valid quantity. Thus, we need to quantify such tweets by taking an assumption that the average family size is 5. Similarly, in the tweet "plz somebody come and save us abt 75 houses submerged in paraniputhur chennai near mangadu no water food plz save us", again we have to assume that one family of 5 persons on average resides in each house; thus, 75 houses mean person count is 375 .

- In some tweets like "100 girls, 200 boys stranded in Lalaji Memorial Omega School, Kolapakkam, Chennai. water level $>6 \mathrm{ft}$ rising, urgent help reqd", we need to sum the multiple quantities stated, to get the actual person count.

- In tweets like "Please send boat to rescue 20 to 30 employees of Madras Fertilizers Limited gate at Manali Chennai-68. Emergency rescue needed ...so plz help", the person count is mentioned in a range. In such cases, we consider the maximum value as count.

- In the tweet "A train from Kerala is coming today with 3 tons of food items but lack of volunteers to distribute the goods in Chennai", ' 3 tons' needs to be converted to person count (1200) by considering the fact that research shows that most humans eat between three to five pounds of food per day.

- A single tweet may indicate multiple resource-needs or availabilities; in such cases, a single tweet can result in multiple need-tuples or availability-tuples. For instance, consider the tweet "More than 300 people stranded at Greenways Rd MRTS Stn... Requires help/food/water/ bedsheets etc \#chennairains". Since this tweet informs about multiple resource-needs, multiple need-tuples are generated. Thus, we have observed several challenges associated with the automatic extraction of tuples from microblogs (tweets) for resource allocation. Hence, we employ a semi-automatic approach to construct the final need-tuples and availability-tuples from the tweets, where a human looks over the tuples identified automatically by the parser (described earlier in this section), and correct the tuples if necessary. Tables 2 and 3 give examples of need-tweets and availability-tweets, and the corresponding need-tuples and availability-tuples, identified by this methodology from the corresponding tweets.

The next section presents our model for resource allocation based on the need-tuples and availability-tuples extracted from microblog streams.

\section{Frameworks for resource allocation}

As stated earlier, our objective is to develop a framework for real-time resource allocation in a post-disaster situation. Hence, we are using microblogs as the information source for the present work, since microblogs are known to be important sources of real-time information. However, 
Table 2 Examples of need-tweets and corresponding need-tuples \{Resource, Location, Quantity, Emergency score\}

\begin{tabular}{|c|c|}
\hline Need-tweet & Extracted need-tuples \\
\hline $\begin{array}{l}\text { Need food/blankets for } 50 \text { people stranded in Chinmaya Vidyalaya, } \\
\text { Virugambakkam }\end{array}$ & $\begin{array}{l}\{\text { Food, 'Chinmaya Vidyalaya, Virugambakkam', } 50,10\} \text { \{Blankets, } \\
\text { 'Chinmaya Vidyalaya, Virugambakkam', } 50,10\}\end{array}$ \\
\hline $\begin{array}{l}\text { Food + aid needed. } 200 \text { ppl stuck at factory in No.6 GST Road, } \\
\text { Guduvanchery, Chennai }\end{array}$ & $\{$ Food, '6 GST Road, Guduvanchery, Chennai', 200, 10\} \\
\hline $\begin{array}{l}700 \text { people are struggling without food in Nandambakkam Canton- } \\
\text { ment kalyana Mandapam }\end{array}$ & $\{$ Food, 'Nandambakkam Cantonment kalyana Mandapam', 700, 10\} \\
\hline $\begin{array}{l}70 \text { people stuck on rooftop c-9,TVK Industrial estate,Guindy,Chennai } \\
32 \mathrm{Pls} \text { help }\end{array}$ & $\begin{array}{l}\text { \{Logistics, 'rooftop c-9, TVK Industrial state, Guindy, Chennai 32', 70, } \\
10\}\end{array}$ \\
\hline $\begin{array}{l}\text { plz somebody come and save us abt } 75 \text { houses submerged in paranipu- } \\
\text { thur chennai near mangadu no water food plz save us }\end{array}$ & $\{$ Food, 'paraniputhur chennai near mangadu', 375,10$\}$ \\
\hline $\begin{array}{l}\text { Pls arrange rescuing } 6 \text { mths pregnant and } 2 \text { elderly from Jaffarkhanpet } \\
\text { [mobile no] }\end{array}$ & $\{$ Logistics, Jaffarkhanpet, 3, 20\} \\
\hline $\begin{array}{l}\text { Hey chennai memes a family is stuck near that kasi thestre they have a } \\
11 / 2 \text { year old kid! }\end{array}$ & $\{$ Logistics, kasi thestre, 5,20$\}$ \\
\hline
\end{tabular}

Emergency score is higher (20) in those cases where children/senior citizens/pregnant women need help

Table 3 Examples of availability-tweets and corresponding availability-tuples \{Resource, Location, Quantity\}

\begin{tabular}{|c|c|}
\hline Availability-tweet & Extracted availability-tuples \\
\hline $\begin{array}{l}\text { Cheer NGO can provide shelter,food for } 50 \mathrm{ppl} \text { Address is No.21/C, Taylors } \\
\text { Road, Kilpauk }\end{array}$ & $\begin{array}{l}\{\text { Food, 'No.21/C, Taylors Road, Kilpauk', 50\}, \{Shelter, } \\
\text { 'No.21/C, Taylors Road, Kilpauk', 50\} }\end{array}$ \\
\hline $\begin{array}{l}\text { food for } 300 \mathrm{ppl} \text {, Saritha, Besant Nagar - [mobile no] Ayyapn [mobile no] for } \\
\text { grocery } \mathrm{n} \text { food }\end{array}$ & $\{$ Food, 'Saritha, Besant Nagar', 300$\}$ \\
\hline Gopal ([mobile no] can accomodate $5 \mathrm{ppl}$ in Thiruvanmiyur & $\{$ Shelter, Thiruvanmiyur, 5$\}$ \\
\hline $\begin{array}{l}\text { Anybody need accomdation in Chennai Central. can accommodate } 15 \mathrm{ppl} \\
\text { and provide food and shelter }\end{array}$ & $\{$ Food, Chennai Central, 15$\},\{$ Shelter, Chennai Central,15\} \\
\hline $\begin{array}{l}\text { Have a school } 2 \text { accmdate } 75-100 \text { in Nungambakkam.food at Chetpet } 2 \\
\text { closely around } 1000 \mathrm{ppl}+\end{array}$ & $\{$ Food, Nungambakkam, 1000$\},\{$ Shelter, Nungambakkam, 100\} \\
\hline $\begin{array}{l}20 \text { divers, } 11 \text { boats rushed to Chennai for relief operations on board INS } \\
\text { AIRAVAT! }\end{array}$ & $\{$ Divers, Chennai Port, 20$\}\{$ Boats, Chennai Port, 11$\}$ \\
\hline
\end{tabular}

this decision of using microblogs brings in some challenges, as follows. Information of the need/availability of a particular resource at a particular location may be available only intermittently on social media, e.g. at the gap of several days (and not regularly on a daily basis, as assumed in the prior work (Basu et al. 2018b)). Also, we have noticed that resource-needs often come up at new locations, for instance, due to aftershocks of an earthquake, or a new area getting flooded. Similarly, resources may also become available at new locations dynamically, which can then be used to satisfy needs at nearby locations. Hence, when dealing with information streams collected dynamically from social media, it is not practical to assume a fixed set of shelter-points and warehouses, as done in (Basu et al. 2018b). Hence, we adapt the model in (Basu et al. 2018b) for our situation. This section describes our proposed postdisaster resource allocation framework in detail.

\subsection{Proposed resource allocation framework utilizing microblogs}

We assume the following working of our proposed framework, that is illustrated in Fig. 1. On a certain day in the aftermath of a disaster event (say, day $t$ ), need-tweets and availability-tweets are identified from the stream of tweets posted on the said day, e.g. by using the methodologies developed in as (Basu et al. 2017a, 2019; Khosla et al. 2017). Then, the need-tweets and availability-tweets are parsed to obtain the need-tuples and availability-tuples as described in Sect. 3. These need-tuples and availability-tuples are fed into the resource allocation framework (which is the primary focus on this present paper). The resource allocation framework also takes some other inputs, such as the unfulfilled needs and residual availabilities from the previous day (day $t-1)$, a deployment time 


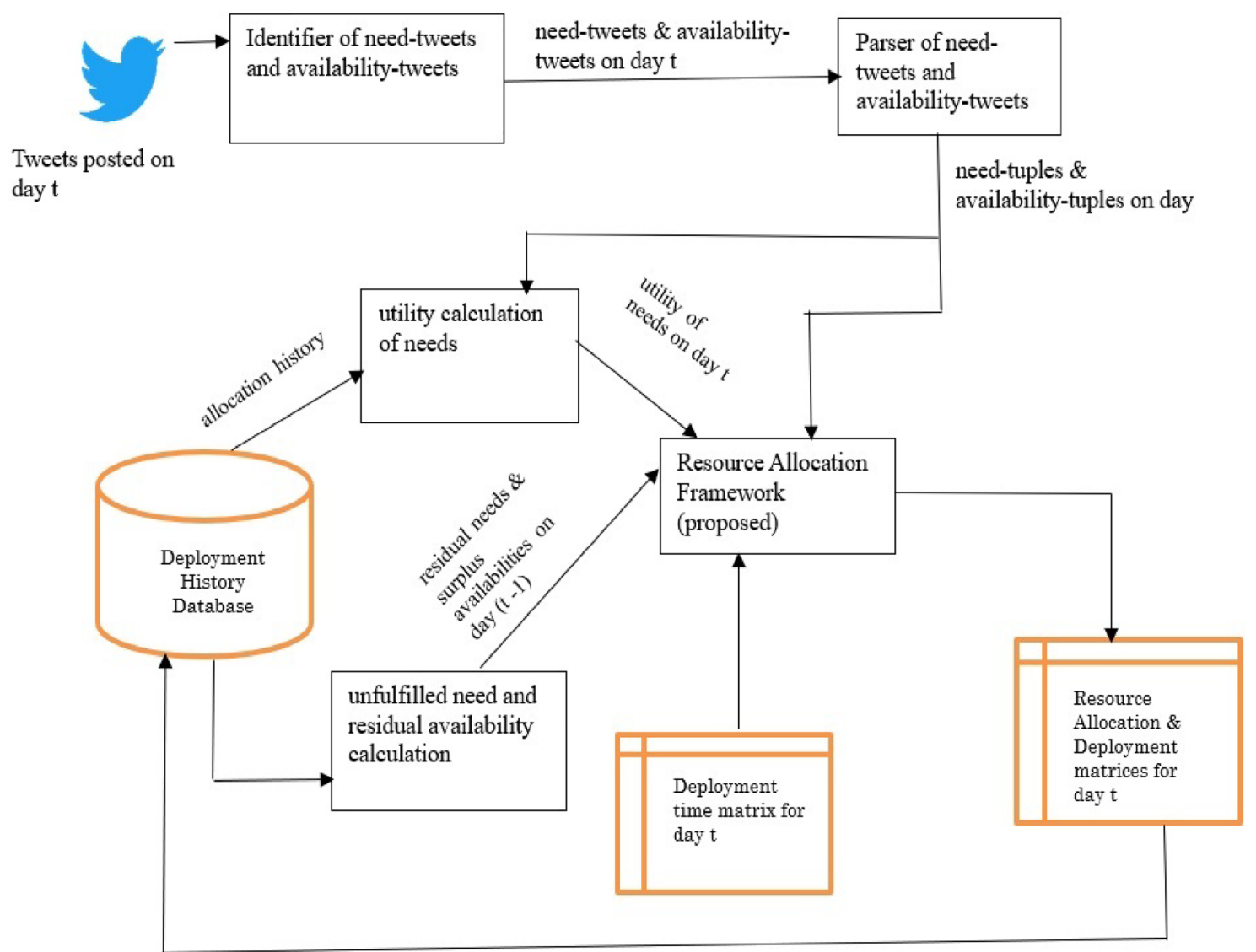

Fig. 1 An illustration of the functioning of our proposed framework for optimized real-time resource allocation in a post-disaster scenario

matrix that states the time needed to transport resources between various locations, and so on.

Additionally, the resource allocation framework considers the utility of various resource-needs, based on the deployment history (that is assumed to be stored in a database). Intuitively, the utility represents the priority with which a resource is needed at a particular need-location. If the said location has been receiving a regular supply of the resource in the prior days, the utility of this need would be low; conversely, if the said location has not received the resource for several days, the utility of this need will be high. The concept of utility of resource-needs, and other details such as the deployment time matrix will be explained in the latter parts of this section.

Based on all these information, the resource allocation framework will suggest some resource deployments for day $t$ (the resource allocation and deployment matrices-to be explained later). We assume that these deployments are carried out on the same day. The deployments are also stored in the deployment history database for future use.

On the next day (day $t+1)$, the newly emerging needs and availabilities are parsed from the tweets posted on the said day. The unfulfilled needs and residual availabilities from day $t$ are added to the newly emerging needs and availabilities (that come up on day $t+1$ ) and again fed to the resource allocation module for suggesting resource deployments on day $t+1$. In this way, the process will continue.

The rest of this section describes the framework for optimal allocation of resources in detail. All the symbols used to describe the model are listed in Table 4. We start by summarizing the model developed in (Basu et al. 2018b) in Sect. 4.2 and then describe our modifications to the model in Sect. 4.3.

\subsection{Overview of the model by Basu et al. (2018b)}

In this section, we give an overview of the model in (Basu et al. 2018b).

\subsubsection{Utility of resources}

Motivated by the notion of utility defined in classical Economics (McConnell et al. 2014), the utility $u_{r p}$ of a resource $r$ at a location $p$ at a specific point of time is defined as its priority to satisfy the requirement at that location. Note that, demand and utility of a resource are different in that the 
Table 4 Description of the symbols used in our model

\begin{tabular}{ll}
\hline Symbol & Description \\
\hline$P$ & Number of need-locations, i.e. distinct location references present in need-tweets on a particular day \\
$Q$ & Number of availability-locations/warehouses, i.e. number of distinct locations present in availability-tweets on a particular day \\
$R$ & Number of resources considered (e.g. Food, Shelter, Logistic, Health, Cash, as prescribed by UNOCHA) \\
$\mathrm{d}_{\mathrm{rp}}(\mathrm{t})$ & Number of units of resource $r$ needed at need-location $p$, as reported on day $t$ \\
$\mathrm{D}_{\mathrm{rp}}(\mathrm{t})$ & Cumulative number units of resource $r$ needed at need-location $p$, as on day $t$ \\
$\mathrm{u}_{\mathrm{rp}}$ & Utility of resource $r$ at location $p$ \\
$\mathrm{a}_{\mathrm{rq}}(\mathrm{t})$ & Number of available units of resource $r$ at availability-location/warehouse $q$, as reported on day $t$ \\
$\mathrm{~A}_{\mathrm{rq}}(\mathrm{t})$ & Cumulative number of available units of resource $r$ at availability-location $q$, as on day $t$ \\
$\mathrm{~A}_{\mathrm{r}}(\mathrm{t})$ & Total number of units of resource $r$ available as on day $t\left(=\sum_{q=1}^{Q} A_{r q}(t)\right)$ \\
$\mathrm{t}_{\mathrm{rpq}}$ & Time required to deploy each unit of resource $r$ to need-location $p$ from warehouse $q$ \\
$\mathrm{c}_{\mathrm{r}}$ & Capacity of each consignment of resource $r$, i.e. the maximum number of units of $r$ that can be shipped in a single consignment \\
$\mathrm{x}_{\mathrm{rp}}(\mathrm{t})$ & Effective number of units of resource $r$ allocated to need-location $p$ till day $t$ \\
$\mathrm{x}_{\mathrm{trp}}$ & Number of units of resource $r$ allocated to need-location $p$ on day $t$ \\
$\mathrm{y}_{\mathrm{trpq}}$ & Number of units of resource $r$ allocated to need-location $p$ from warehouse $q$ on day $t$ \\
$n_{r}$ & Total number of days of prior allocation for resource $r$ (prior to the present day) \\
\hline
\end{tabular}

demand is the quantity of the resource needed, whereas the utility is the exigency of the demand. In accordance with the classical theory of utility (McConnell et al. 2014), the utility $u_{r p}$ of a resource is expressed as a function of its effective allocation $x_{r p}$ as

$u_{r p}=f\left(x_{r p}\right)$

where $f()$ is some suitable utility function. Intuitively, the present utility of a resource (at a certain location) depends on how much of the resource has already been allocated at this location, and how recently. Hence, utility is a function of allocation.

Now, to derive a general mathematical expression for utility functions, prior works used curve fitting techniques on some conventional utility data sets (utility-clams 2016; utility-marginal 2016; McConnell et al. 2014; Gershuny 2009; Kuo and Liao 2007), to identify the following rule that fits best as a utility function:

$u_{r p}=f\left(x_{r p}\right)=a x_{r p}^{3}+b x_{r p}^{2}+c x_{r p}+d$

where the values of parameters $a, b, c, d$ are experimentally obtained for a given situation. Specifically, for the context of resources in a post-disaster scenario, Basu et al. (Basu et al. 2018b) used the least square method for curve fitting on data of a particular disaster event (floods in the Uttarakhand state of India) to estimate the following values: $a=0.1086, b=-4.122, c=39.97$, and $d=-0.0115$. We assume the same value for these parameters in this work.

Thus, following the classical theory of Economics, Eq. 2 describes utility of a resource $r$ at a specific location $p$ as a function of only its prior effective allocation $x_{r p}$ (at the said location). However, since in a post-disaster scenario, resources are allocated to different locations at non-uniform time intervals, the effective allocation $x_{r p}$ varies with time, i.e. $x_{r p}$ is a function of time $t$. Thus, the utility of a resource $\left(u_{r p}\right)$ is considered as function of both the allocation and time, as described in Eq. 3.

$u_{r p}(t)=f\left(x_{r p}\right)=f(g(t))$

Since it has been observed that, in a post-disaster scenario, resources are allocated at different locations at non-uniform time points $t_{1}, t_{2}, \ldots$, thus Basu et al. (2018b) employed Lagrange's Interpolation formula to find the dependence of the effective allocation $\left(x_{r p}\right)$ on $t$ as represented by Eq. 4 .

$x_{r p}(t)=\sum_{i=1}^{n_{r}} \prod_{j=1, j \neq i}^{n_{r}} \frac{\left(t-t_{j}\right)}{\left(t_{i}-t_{j}\right)} x_{i r p}$

where $x_{\text {irp }}$ is the number of units of resource $r$ that was allocated to location $p$ on day $i$, and $n_{r}$ is the number of days of prior allocation of resource $r$ (to the said location), prior to the present day. Hence, combining Eqs. 2 and 4, Basu et al. proposed a model to calculate utility of a resource at a particular location $p$ at time-instance $t$, considering prior allocation of the same resource at the same location.

For the present study, we have adapted this model to calculate utility of a resource at a particular location. Our modification will be described later in Sect. 4.3.1.

\subsubsection{Integer Programming Models for Optimal Resource Allocation}

Basu et al. (2018b) formulated a strategy to optimize resource allocation using integer programming. They formulated two 
strategies-one for minimization of overall resource deficit, and the other for minimization of resource deployment time-for deciding the resource allocation $x_{r p}$ on day $t$, for all resource $r$ and need-location $p$. We briefly describe these two strategies.

(1) Model for minimizing overall resource deficit The objective of this model is to minimize the overall resource deficit which is formulated as a weighted ratio of unfulfilled demand and the actual demand, with utility as the weight. The objective function of the optimization is follows:

$$
\begin{aligned}
\text { Minimize } & \sum_{p=1}^{P} \sum_{r=1}^{R} \frac{u_{r p}(t)\left(d_{r p}(t)-x_{t r p}\right)}{d_{r p}(t)} \\
& \text { subject to } \\
& \sum_{p=1}^{P} x_{t r p}<A_{r}(t), r=1, \ldots, R, p=1, \ldots, P \\
& x_{t r p} \leq d_{r p}(t), r=1, \ldots, R, p=1, \ldots, P \\
& x_{t r p} \geq 0, r=1, \ldots, R, p=1, \ldots, P
\end{aligned}
$$

where the symbols are as explained in Table 4, and $u_{r p}(t)$ is computed as described above. The solution of this optimization problem gives the values of $x_{t r p}$, i.e. how much of resource $r$ is to be allocated to needlocation $p$ on day $t$. The $x_{t r p}$ values for all resources and locations are arranged in the form of a matrix that is known as the resource allocation matrix for day $t$ each row of this matrix represents a location, and each column represents a resource.

(2) Model for Minimizing Total Resource Deployment Time The previous model determines the optimal amount $x_{r p}$ of resource $r$ that should be allocated to need-location $p$ on day $t$, for every $r$ and every $p$. This model goes further to determine from which warehouse/availabilitylocation(s) this amount of $r$ would be transmitted to $p$, so that the total resource deployment time is minimized. Let $y_{\text {trpq }}$ be the number of units of resources $r$ that is to be deployed/transported to need-location $p$ from the availability-location $q$, on day $t$. Also, let $t_{r p q}$ be the time required to deploy each unit of resource $r$ to need-location $p$ from availability-location $q$. The $t_{r p q}$ values need to be provided as input to the model. Then the total resource deployment time is minimized as follows:

$$
\begin{aligned}
\text { Minimize } & \sum_{r=1}^{R} \sum_{p=1}^{P} \sum_{q=1}^{Q} t_{r p q} *\left\lceil\frac{y_{t r p q}}{c_{r}}\right\rceil \\
& \text { subject to } \\
& \sum_{q=1}^{Q} y_{t r p q}=x_{t r p}, r=1, \ldots, R, p=1, \ldots, P \\
& \sum_{p=1}^{P} y_{t r p q} \leq A_{r q}(t), r=1, \ldots, R, p=1, \ldots, P
\end{aligned}
$$

Here, $c_{r}$ is the number of units of resource $r$ that can be transported at a time (i.e. in a single consignment) from availability-location $q$ to need-location $p$. The rest of the symbols are as explained in Table 4. The solution to this optimization problem gives the values of $y_{\text {trpq }}$ which says what amount of a resource needs to be transported on day $t$, and from which availabilitylocation to which need-location.

\subsection{Adapting the model in Basu et al. (2018b) for real-time resource allocation}

In this section, we describe how we adapt the model (Basu et al. 2018b) for real-time resource allocation, where microblogging streams are used for extracting resource-needs and resource-availabilities. This adaptation of the model is necessary to account for the uncertainty and incompleteness of information available from social media.

\subsubsection{Computing utility of resources from incomplete allocation information}

Similar to (Basu et al. 2018b), we also consider the utility $u_{r p}(t)$ of resource $r$ at location $p$ on day $t$ to be a function of the prior allocation $x_{r p}$ of the resource at the said location. The prior work (Basu et al. 2018b) used allocation data obtained from a Government portal, which was collected over several months after the actual disaster event. These data were organized and complete and contained allocation data for each day during a $n$-day period after the disaster event. Hence, it was feasible to apply Eq. 4 which needs allocation data of a particular resource at a particular location for at least two distinct days ( $t_{i}$ and $t_{j}$ in Eq. 4).

In contrast, when microblog streams are used as the information source (as in the present work), it is often seen that allocation data are very sparse and incomplete, and new need-locations / resources (with no prior allocation) come up dynamically. Thus, Eq. 4 needs to be adapted to this dynamic environment. To this end, we employ three distinct equations for computing utility of resources $u_{r p}$-Eqs. 7, 8, and 9). On a day when the demand $d_{r p}$ for the resource $r$ is reported for 
the first time at location $p$, utility $\left(u_{r p}\right)$ is calculated using Eq. 7:

$u_{r p}(t)=d+$ Emergency_score

On a day on which the demand of the resource $r$ at location $p$ is reported for the second time, utility is calculated using Eq. 8 .

$$
\begin{aligned}
u_{r p}(t)= & a\left(\sum_{i=1}^{n_{r}}\left(t-t_{j}\right) x_{i r p}\right)^{3}+b\left(\sum_{i=1}^{n_{r}}\left(t-t_{j}\right) x_{i r p}\right)^{2} \\
& +c\left(\sum_{i=1}^{n_{r}}\left(t-t_{j}\right) x_{i r p}\right)+d+\text { Emergency_score }
\end{aligned}
$$

Finally, if the demand of $r$ at $p$ has been reported on two or more days prior to day $t$, utility is calculated using Eq. 9 (which is what is used by Basu et al. (2018b)).

$$
\begin{aligned}
u_{r p}(t)= & a\left(\sum_{i=1}^{n_{r}} \prod_{j=1, j \neq i}^{n_{r}} \frac{\left(t-t_{j}\right)}{\left(t_{i}-t_{j}\right)} x_{i r p}\right)^{3} \\
& +b\left(\sum_{i=1}^{n_{r}} \prod_{j=1, j \neq i}^{n_{r}} \frac{\left(t-t_{j}\right)}{\left(t_{i}-t_{j}\right)} x_{i r p}\right)^{2} \\
& +c\left(\sum_{i=1}^{n_{r}} \prod_{j=1, j \neq i}^{n_{r}} \frac{\left(t-t_{j}\right)}{\left(t_{i}-t_{j}\right)} x_{i r p}\right) \\
& +d+\text { Emergency_score }
\end{aligned}
$$

Additionally, as stated earlier, we also consider an emergency score depending on the criticality of resource being considered. For instance, if the resource being considered is needed by children/pregnant women/senior citizens, then the emergency score is higher than otherwise.

\subsubsection{Handling unfulfilled needs and surplus availabilities}

On each day $t$, we use the integer programming-based optimization framework (Eqs. 5 and 6) for deciding the optimal amount of resources to be allocated to specific need-locations, and for deciding the availability-location/warehouse from which the resources should be allocated. However, it needs to be noted that the availability of a resource on day $t$ may be insufficient to fulfill all demands of the resource on that day; thus, the unfulfilled demands should be carried over to the next day $t+1$. Similarly, a resource may be available in surplus quantity on day $t$, and the surplus amount (after fulfilling the demands as on day $t$ ) should be carried over to the next day $t+1$ in order to potentially fulfill future demands for the resource. These considerations are not included in the model developed by Basu et al. (2018b). Hence, we include the following modifications in the existing model.
Let $d_{r p}(t)$ be the number of units of resource $r$ that is reported to be freshly needed at need-location $p$ on day $t$. In other words, these resource-needs are extracted from the need-tweets posted on day $t$. We denote $D_{r p}(t)$ to be the cumulative need of $r$ at $p$ on day $t$, taking into account the unfulfilled need on the previous day (if any) and fresh needs reported on day $t$ (if any). Thus, we can compute the cumulative demand on day $t+1$ as

$D_{r p}(t+1)=D_{r p}(t)-x_{t r p}+d_{r p}(t+1)$

where $x_{t r p}$ is the quantity of resource $r$ allocated to location $p$ on day $t$ (as computed using Eq. 5).

Similarly, let $a_{r q}(t)$ be the number of units of resource $r$ that is reported to be freshly available at availability-location $q$ on day $t$, as extracted from the availability-tweets posted on day $t$. We denote $A_{r q}(t)$ to be the cumulative availability of $r$ at $q$ on day $t$, taking into account the surplus availability on the previous day (if any) and fresh availability reported on day $t$ (if any). Thus, we have

$A_{r q}(t+1)=A_{r q}(t)-\sum_{p=1}^{P} y_{t r p q}+a_{r q}(t+1)$

where $y_{\text {trpq }}$ is the quantity of resource $r$ that was allocated to need-location $p$ from availability-location $q$ on day $t$, as computed by Eq. 6. Thus, the sum in Eq. 11 is meant to compute the total amount of $r$ that was allocated from location $q$ to all need-locations on day $t$.

\subsubsection{Handling new locations and resources}

In a disaster scenario, the situation can change dynamically in practice. For instance, new demands and availabilities of resources can evolve (Basu et al. 2017b). For instance, consider that after an earthquake, the need of food and shelter is prevalent on day $t-1$ at several locations. On day $t$, there can be an aftershock of the earthquake affecting new locations, which also subsequently need such resources. Again, various external agencies start sending relief materials to the disaster-struck region, and hence various resources can become available at new locations. Thus, new availabilitylocations (warehouses) can come into picture. In fact, the set of resources needed/available also changes with time, as observed in our prior study (Basu et al. 2017b). The model by Basu et al. (2018b) does not consider such changes in the set of need-locations/availability-locations/resources. Hence, we have modified the model to consider these practical dynamicity of a disaster situation.

In our modified model, the values of $P$ (number of needlocations), $Q$ (number of availability-locations) and $R$ 
Table 5 Mapping of location and resources from tweets posted during the Chennai floods on 2 December, 2015 (which we consider Day 1)

\begin{tabular}{|c|c|c|}
\hline & Location & Resource, Quantity \\
\hline \multicolumn{3}{|l|}{ Need-Tweet (extract) } \\
\hline $\begin{array}{l}\text { More than } 300 \text { people stranded at Greenways Rd MRTS Stn... Requires help food } \\
\text { water bedsheets etc \#chennairains }\end{array}$ & Greenways Rd (P1) & Food (R1): 300, Shelter (R2): 300 \\
\hline $\begin{array}{l}\text { Need food/blankets for } 50 \text { people stranded in Chinmaya Vidyalaya, Virugambak- } \\
\text { kam \#chennairains \#chennaifloods \#chennairainshelp }\end{array}$ & Virugambakkam (P2) & Food (R1): 50, Shelter (R2): 50 \\
\hline $\begin{array}{l}700 \text { people are struggling without food in Nandambakkam Cantonment kalyana } \\
\text { Mandapam \#chennairains \#Chennai \#chennaifloods }\end{array}$ & Nandambakkam (P3) & Food (R1): 700 \\
\hline $\begin{array}{l}\text { plz somebody come and save us abt } 75 \text { houses submerged in paraniputhur chennai } \\
\text { near mangadu no water food plz save us }\end{array}$ & Magadu (P4) & Food(R1): 375 \\
\hline \multicolumn{3}{|l|}{ Availability-Tweet (extract) } \\
\hline $\begin{array}{l}\text { Cheer NGO can provide shelter food for } 50 \text { ppl Address is No.21/C, Taylors Road, } \\
\text { Kilpauk, Chennai - 10. [mobile no.] }\end{array}$ & Kilpauk (Q1) & Food (R1): 50, Shelter (R2): 50 \\
\hline $\begin{array}{l}\text { Have a school } 2 \text { accmdate } 75-100 \text { in Nungambakkam. food at Chetpet } 2 \text { closely } \\
\text { around } 1000 \mathrm{ppl} \text { [mobile no.] \#chennairains \#Chennai }\end{array}$ & Nungambakkam (Q2) & Food (R1): 1000, Shelter (R2): 100 \\
\hline
\end{tabular}

We get the information of two resources (R1, R2), four need-locations (P1, P2, P3, P4), and two availability-locations (Q1 and Q2). The quantities are in the unit of persons. Note that, in the last need-tweet, a 'house' is assumed to comprise of 5 persons on average

(number of resource classes) can actually be different on different days. ${ }^{3}$ New need-locations may be introduced on a certain day (day $t$ ), e.g. due to new areas being flooded, or due to aftershocks of an earthquake affecting new locations. For such a new need-locations $p^{\prime}$, the utility of resource $r$ will be calculated from Eq. 7, i.e. considering $x_{r p^{\prime}}(t-1)=0$. The cumulative demand matrix will be updated with the entries of the demand tuples from previously encountered locations as well as the new location $\left(p^{\prime}\right)$ and the utility matrix will be updated accordingly. Note that, the dimensions of the cumulative demand matrix, resource allocation matrix, etc., may increase as new locations are encountered (or if new types of resource are introduced).

Summary of section We first gave a high-level description of our proposed resource allocation framework utilizing information posted on microblogging sites (in Sect. 4.1). Figure 1 gives an illustrative overview of our proposed resource allocation framework. Then, we described the model developed in (Basu et al. 2018b) in Sect. 4.2. This model assumed complete data regarding daily resource needs and availabilities at a fixed set of locations, which can only be available several months after a disaster event, and not in real time. Since our objective is real-time resource allocation, we need to rely on uncertain and incomplete data available on social media. Hence, we modify the model to adapt to our needs, and we describe our modifications to the model in Sect. 4.3.

\footnotetext{
3 Technically, we should have made these quantities functions of time, i.e. $P(t), Q(t), R(t)$ should have been used to denote the respective quantities on day $t$. But we misuse the notations for simplicity.
}

Next, we proceed to perform experiments to evaluate the proposed model and to compare it with that of some baseline resource allocation models.

\section{Experiments and analysis}

We now demonstrate the working of our resource allocation model over a working example. We also compare our model with some baseline models that have been designed for a similar setting, using the same working example.

\subsection{A working example}

To illustrate the experimental results, we consider a small example derived from some of the tweets posted during the severe floods in December 2015 in the Indian city of Chennai (the same disaster event from which examples in Sect. 3 were given). We consider two sets of tweets

(1) Need-tweets and availability-tweets posted on 02 December, 2015, which we consider as Day 1; the tweets are shown in Table 5, and

(2) Need-tweets and availability-tweets posted on 03 December, 2015, which we consider as Day 2; these tweets are shown in Table 6. From these tweets, we get the need and availability of two resources 'Food' and 'Shelter' (which we refer to as R1 and R2) in four needlocations (which we refer to as P1, P2, P3, and P4) and two warehouses/availability-locations (which we refer to as Q1 and Q2), over two days. 
Table 6 Mapping of location and resources from tweets posted during the Chennai floods on 3 December, 2015 (which we consider Day 2)

\begin{tabular}{|c|c|c|}
\hline & Location & Resource, Quantity \\
\hline \multicolumn{3}{|l|}{ Need-Tweet (extract) } \\
\hline $\begin{array}{l}\text { Greenways Road, RK Puram Road \#Chennai- patients, doctors staff stranded - no } \\
\text { food. Over 100ppl stranded \#ChennaiRescue }\end{array}$ & Greenways Road (P1) & Food (R1): 100 \\
\hline $\begin{array}{l}1000 \text { ppl stranded @ Virugambakkam require food and shelter-[mobile no.]-veri- } \\
\text { fied \#ChennaiFloods }\end{array}$ & Virugambakkam (P2) & Food(R1): 1000 , Shelter(R2): 1000 \\
\hline $\begin{array}{l}\text { Need bedding and clothing for } 180 \text { people near Nandambakkam Panchayat Water } \\
\text { Tank. Any help guidance? }\end{array}$ & Nandambakkam (P3) & Shelter(R2): 180 \\
\hline $\begin{array}{l}\text { Help ASAP } 75 \text { ppl stuck in, Mangadu Rd, Kovur, Rajappa Nagar No food and } \\
\text { water ,complete power shutdown in building. }\end{array}$ & Mangadu(P4) & Food(R1): 75 \\
\hline \multicolumn{3}{|l|}{ Availability-Tweet (extract) } \\
\hline $\begin{array}{l}\text { \#Chennai Food Availability For } 1500 \text { People and shelter for 50!! Kilpauk:[mobile } \\
\text { no] }\end{array}$ & Kilpauk(Q1) & Food(R1): 1500, Shelter(R2): 50 \\
\hline $\begin{array}{l}\text { \#PrayForChennai Foods Ready For } 50 \mathrm{ppl} \text { and } 900 \text { sleeping bags are available in } \\
\text { Chennai Nungambakkam Contact - [mobile no] }\end{array}$ & Nungambakkam (Q2) & Food (R1): 50, Shelter (R2): 900 \\
\hline
\end{tabular}

We get the information of two resources (R1, R2), four need-locations (P1, P2, P3, P4), and two availability-locations (Q1 and Q2). The quantities are in the unit of persons

Table 7 Example need matrix for Day 1-gives the number of units of the resources (R1, $\mathrm{R} 2)$ that are needed in the four need locations (P1, P2, P3, P4) on Day 1

\begin{tabular}{lrr}
\hline & R1 & R2 \\
\hline P1 & 300 & 300 \\
P2 & 50 & 50 \\
P3 & 700 & 0 \\
P4 & 375 & 0 \\
\hline
\end{tabular}

The entries are derived from the need-tweets in Table 5

\begin{tabular}{lrr}
\hline & R1 & R2 \\
\hline Q1 & 50 & 50 \\
Q2 & 1000 & 100 \\
\hline
\end{tabular}

The entries are derived from the availability-tweets in Table 5
Table 9 Example need matrix for Day 2-gives the number of units of the resources (R1, $\mathrm{R} 2)$ that are needed in the four need locations (P1, P2, P3, P4) on Day 2

Table 10 Example availability matrix for Day 2-gives the number of units of the resources $(\mathrm{R} 1, \mathrm{R} 2)$ that are available in the two availability locations (Q1, Q2) on Day 2 (Q1, Q2) on Day 1

Table 8 Example availability matrix for Day 1-gives the number of units of the resources $(\mathrm{R} 1, \mathrm{R} 2)$ that are available in

tocations


$\mathrm{P} 2)$. Table 11 shows the deployment time matrix, that gives the time required to transport one unit of resource from one location to the other locations.

We make some simplifying assumptions-we assume that the deployment times remain constant over both Day 1 and Day 2. Also, we assume that each unit of a resource is transported individually as a consignment, i.e. $c_{r}=1$ for both resources $\mathrm{R} 1$ and $\mathrm{R} 2$.

\subsection{Baseline approaches for resource allocation}

The setting used by Basu et al. (2018b) is completely different from our setting, since they used historical government reports collected several months after a disaster event (disaster-uttarakhand 2013); hence, as simulation environment, they considered a fixed set of need-locations and availability-locations (warehouses). However, the present model considers the practical scenario of dynamically changing need-locations and availability-locations; hence, it would not be fair to compare the performance of the model used by Basu et al. (2018b).

To compare the performance of our methodology, we consider the following two baselines in a similar setting:

(1) Baseline 1: Simple baseline this is a naive methodology that considers an availability-tweet to match a needtweet if both are referring to the same resource. Thus, this baseline does not consider locations.

(2) Baseline 2: Baseline based on location proximity here we consider the methodology of our prior work (Dutt et al. 2019) for matching need-tweets and availabilitytweets by considering resource-similarity, as well as geographical-proximity of the locations of resource need and availability. To compute the geographicalproximity score, the geographical coordinates of the need-location and availability-location (that are extracted from the need-tweet and availability-tweet, respectively) are considered, and the distance between the two locations is obtained using Google Maps. This distance is normalized using a bounding box around the region where the disaster has occurred (e.g. a bounding box around Nepal for the Nepal earthquake, or a bounding box around the Indian city of Chennai for the 2015 Chennai floods). Tweets are ranked according to a linear combination of the resource-similarity score and the geographical-proximity score (see (Dutt et al. 2019) for details). As reported in (Dutt et al. 2019), a need-tweet and an availability-tweet are considered as matched if (i) they mention about the need and availability of the same resource, and (ii) the road-distance between the need-location and the availability-location is less than a certain threshold. Note that, according to both these baselines, each need-tweet/availability-tweet is considered in isolation. Hence, a particular availability-tweet can be matched to multiple need-tweets. In contrast, the model proposed in the present work considers all needs and availabilities together and attempts to optimize resource allocations accordingly. Thus, the comparison of these baselines with the model proposed in this present work is meant to highlight the need for considering the optimization framework described earlier.

\subsection{Evaluation metric}

We will next compare the performance of various resource allocation models on the working example. To this end, we consider the following performance metrics.

\subsubsection{Shelter-specific resource deficit (SRD)}

We consider the $\operatorname{SRD}(t)$ metric introduced by Kondaveti and Ganz (2009) to evaluate the rate at which resource deficits are minimized at each need-location (with respect to time). For a particular need-location $p$, the metric is defined as follows:

$$
\begin{aligned}
& \operatorname{SRD}_{p}(t)=\frac{1}{\sum_{r=1}^{R} u_{r p}(t)} \\
& \times \sum_{r=1}^{R}\left(u_{r p}(t) \times\left(1-\frac{\sum_{q=1}^{Q} y_{t r p q} \times f_{r p q}\left(t>t_{r p q}\right)}{d_{r p}(t)}\right)\right)
\end{aligned}
$$

Here, $t$ is the time at which SRD is measured and $t_{r p q}$ is the time taken to deploy resource $r$ from availability-location $q$ to need-location $p$. The function $f_{r p q}\left(t>t_{r p q}\right)$ is a step function that takes the value 1 if $t>t_{r p q}$ and 0 otherwise. Note that, since time is an important factor in resource allocation, this metric distinguishes between the following two cases:

Case 1 If the time $t_{r p q}$ taken to deploy a particular resource $r$ from availability-location $q$ to need-location $p$ is greater than the time $t$ (when SRD is being measured), then deployment of that resource $r$ at $p$ is not considered effective. Hence, the step function $f_{r p q}\left(t>t_{r p q}\right)=0$. In this scenario, according to Eq. 12, resource deficit of $r$ at $p$ reaches its maximum. In the extreme case, if this situation occurs for all $R$ resources, then the $\mathrm{SRD}$ at $p$ is maximum, i.e. $S R D_{p}(t)=1$.

Case 2 If the time $t_{r p q}$ taken to deploy resource $r$ from $q$ to $p$ is less than the time $t$ (when SRD is being measured), then the step function $f_{r p q}\left(t>t_{r p q}\right)=1$. In this case, from Eq. 12, it is evident that SRD at $p$ depends on both number of units of resource $r$ allocated to $p$ from $q$ on day $t\left(y_{\text {trpq }}\right)$, as well as the utility of that resource at $p\left(u_{r p}(t)\right)$.

Note that, this metric considers all resources $r$ for a particular need-location $p$ and at time $t$; hence, the expression includes summation over all $R$ number of resources as well 
Table 12 Allocation matrix for day 1, following Simple Baseline-shows the number of units of each resource (R1 and $\mathrm{R} 2$ ) that are allocated to each need-location (P1, P2, P3, P4)

\begin{tabular}{lrr}
\hline & R1 & R2 \\
\hline P1 & 300 & 150 \\
P2 & 50 & 0 \\
P3 & 700 & 0 \\
P4 & 0 & 0 \\
\hline
\end{tabular}

Table 13 Deployment matrix for resource R1 for day 1, following Simple Baselineshows the number of units of resource $\mathrm{R} 1$ that are allocated to each need-location $(\mathrm{P} 1, \mathrm{P} 2$, P3, P4) from each availabilitylocation $(\mathrm{Q} 1, \mathrm{Q} 2)$

\begin{tabular}{lrr}
\hline & Q1 & Q2 \\
\hline P1 & 50 & 250 \\
P2 & 0 & 50 \\
P3 & 0 & 700 \\
P4 & 0 & 0 \\
\hline
\end{tabular}

as summation over all $Q$ availability-locations. Thus, this metric determines the rate at which resource deficits are minimized at each individual need-location $p$.

\subsubsection{Average resource deficit (ARD)}

This metric measures the rate at which overall resource deficit is minimized across all need-locations. $A R D(t)$ is defined as follows:

$\operatorname{ARD}(t)=\frac{\sum_{p=1}^{P} S R D_{p}(t)}{P}$

where $P$ is the total number of need-locations on day $t$.

\subsection{Applying the resource allocation methodologies to the working example}

We now apply the three resource allocation models (the two baseline methods and the proposed model) to the working example described in Sect. 5.1.

\subsubsection{Applying the simple baseline}

According to our simple baseline (that does not consider locations), all warehouses (availability-locations) are effectively considered together, as if there was a single warehouse. Thus, the allocation would effectively start from a particular availability-location/warehouse (say, Q1) and continue as long as there is any resource remaining in the said warehouse. When the resource present in the said warehouse is exhausted, the allocation will start from the next warehouse (say, Q2), and so on.

For day 1, we consider the resource-needs reported in Table 7 and the resource-availabilities reported in Table 8 . Following the simple baseline, Table 12 shows the allocation matrix that gives the number of units of each resource (R1
Table 14 Deployment matrix for R2 for day 1, following Simple Baseline

\begin{tabular}{lrr}
\hline & Q1 & Q2 \\
\hline P1 & 50 & 100 \\
P2 & 0 & 0 \\
P3 & 0 & 0 \\
P4 & 0 & 0 \\
\hline
\end{tabular}

Table 15 Unfulfilled need matrix for day 1 , following Simple Baseline-shows the demand (number of units) of each resource that remains unfilfilled at each need-location (P1, P2, P3, P4), after the allocation is done for Day 1

\begin{tabular}{rrr}
\hline & R1 & R2 \\
\hline P1 & 0 & 150 \\
P2 & 0 & 50 \\
P3 & 0 & 0 \\
P4 & 375 & 0 \\
\hline
\end{tabular}

and $\mathrm{R} 2)$ that are to be allocated to each need-location (P1, P2, P3, P4) on day 1 . The deployment matrices for R1 and $\mathrm{R} 2$ are represented by Tables 13 and 14, respectively; these tables show the number of units of a resource that are to be allocated to each need-location (P1, P2, P3, P4) from each availability-location $(\mathrm{Q} 1, \mathrm{Q} 2)$ on day 1 . After the allocation is done for Day 1, Table 15 illustrates the unfulfilled demand matrix.

Accordingly, the $S R D(t)$ values for each need-location are calculated, and also the Average Resource Deficit $A R D(t)$ value is calculated using Eqs. 12 and 13, respectively. These values (for day 1) are reported later in Table 24, for comparison with those for the other methodologies.

For day 2 , the need matrix is generated by combining the fresh demands encountered on day 2 (Table 9) with the unfulfilled demands from day 1 (Table 15). Similarly, the availability matrix for day 2 is generated by combining the fresh availabilities reported on day 2 (Table 10) with the residual availabilities at the end of day 1 (if any). Then, the allocation matrix and deployment matrices for day 2 are computed by solving the optimization problem described earlier. For brevity, we have not shown the allocation and deployment matrices for day 2 . The values of our evaluation metrics- $S R D(t)$ and $A R D(t)$ - for day 2 are reported later in Table 25, for comparison with those for the other methodologies.

\subsubsection{Applying the baseline based on location proximity}

This baseline, based on our prior work (Dutt et al. 2019), considers both resource similarity and geographical proximity of the location of need and availability, to match the need-tweets and availability-tweets.

Thus, for day 1 (considering the needs shown in Table 7 and availabilities in Table 8), the allocation matrix following this baseline is shown in Table 16. The corresponding deployment matrices for resources R1 and R2 are reported 
Table 16 Allocation matrix for day 1, following Baseline based on location proximity-shows the number of units of each resource (R1 and R2) that are allocated to each need-location (P1, P2, P3, P4)

Table 17 Deployment matrix for R1 for day 1 , following Baseline based on location proximity—shows the number of units of resource R1 that are allocated to each need-location (P1, P2, P3, P4) from each availability-location (Q1, Q2)

\begin{tabular}{lrr}
\hline & R1 & R2 \\
\hline P1 & 50 & 50 \\
P2 & 0 & 0 \\
P3 & 700 & 0 \\
P4 & 300 & 0 \\
\hline
\end{tabular}

\begin{tabular}{rrr}
\hline & Q1 & \multicolumn{1}{c}{ Q2 } \\
\hline P1 & 50 & 0 \\
P2 & 0 & 0 \\
P3 & 0 & 700 \\
P4 & 0 & 300 \\
\hline
\end{tabular}

Table 18 Deployment matrix for R2 for day 1, following Baseline based on location proximity

\begin{tabular}{lrl}
\hline & Q1 & Q2 \\
\hline P1 & 50 & 0 \\
P2 & 0 & 0 \\
P3 & 0 & 0 \\
P4 & 0 & 0 \\
\hline
\end{tabular}

Table 19 Unfulfilled need matrix for day 1 , following Baseline based on location proximity — shows the demand (number of units) of each resource that remains unfulfilled at each need-location (P1, P2, $\mathrm{P} 3, \mathrm{P} 4)$, after the allocation is done for Day 1
Table 20 Allocation Matrix for Day 1, following proposed methodology—shows the number of units of each resource (R1 and R2) that are allocated to each need-location (P1, P2, P3, P4)

Table 21 Deployment Matrix for R1 for Day 1, following proposed methodology-shows the number of units of resource $\mathrm{R} 1$ that are allocated to each need-location (P1, P2, P3, P4) from each availability-location (Q1, Q2)

Table 22 Deployment Matrix for R2 for Day 1, following proposed methodology

\begin{tabular}{lrr}
\hline & R1 & R2 \\
\hline P1 & 300 & 100 \\
P2 & 50 & 50 \\
P3 & 325 & 0 \\
P4 & 375 & 0 \\
\hline
\end{tabular}

\begin{tabular}{lrr}
\hline & Q1 & Q2 \\
\hline P1 & 0 & 300 \\
P2 & 0 & 50 \\
P3 & 0 & 325 \\
P4 & 50 & 325 \\
\hline
\end{tabular}

\begin{tabular}{lrr}
\hline & Q1 & Q2 \\
\hline P1 & 0 & 100 \\
P2 & 50 & 0 \\
P3 & 0 & 0 \\
P4 & 0 & 0 \\
\hline
\end{tabular}

Table 23 Unfulfilled need matrix for day 1 , following proposed optimization modelshows the demand (number of units) of each resource that remains unfulfilled at each need-location (P1, P2, P3, P4), after the allocation is done for

\begin{tabular}{lrr}
\hline & R1 & R2 \\
\hline P1 & 0 & 200 \\
P2 & 0 & 0 \\
P3 & 375 & 0 \\
P4 & 0 & 0 \\
\hline
\end{tabular}
Day 1

in Table 17 and Table 18. Finally, Table 19 shows the unfulfilled needs at each location (shelter) at the end of day 1 .

The process continues on day 2 as explained in Sect. 5.4.1. The total need matrix for day 2 is generated by combining the unfulfilled needs of day 1 and the fresh needs reported on day 2 . The total availability matrix for day 2 is generated by combining the residual availabilities of day 1 and the fresh availabilities reported on day 2 . The allocation for day 2 is done considering the total need matrix and the total availability matrix. We are not stating the details of day 2 for brevity.

\subsubsection{Applying the proposed methodology}

In our proposed model, resources are allocated in an optimized way considering both demand and utility of a resource at a specific need-location. To solve the integer programming model for optimal resource allocation, the LINGO optimization tool is used to generate the allocation and deployment matrices (for each day). ${ }^{4}$ The allocation matrix for day 1 , following the proposed methodology, is shown in Table 20. Tables 21 and 22, respectively, show the deployment plan for the resources R1 and R2 for day 1 . Table 23 shows the unfulfilled resource needs at each need-location at the end of day 1.

The model was applied for day 2 as well, in a similar setting as described for the two baseline methods. We are not reporting the details of day 2 for brevity.

\subsection{Comparing the three resource allocation models}

We now compare the performance of the three resource allocation models (the Simple Baseline, the Baseline based on

\footnotetext{
${ }^{4}$ LINGO: https://www.lindo.com/index.php/products/lingo-andoptimization-modeling.
} 
location proximity, and the proposed methodology) on the working example.

\subsubsection{Reducing resource-deficits}

We use the SRD and ARD metrics that were explained in Sect. 5.3. For a particular model, these metrics are computed using Eqs. 12 and 13 at the end of day 1, considering the resource-allocations on day1 (according to the said model), and again at the end of day 2, considering the resourceallocations prescribed by the said model for day 2 . The SRD and ARD values at the end of day $1(t=1)$ are reported for all three models in Table 24. Similarly, the metrics for day 2 $(t=2)$ are computed and reported in Table 25.

It is evident that the proposed methodology enables deployment of resources in such a way that resource deficit at each need-location is minimized, and the deficit gradually decreases with time. For the present experiment, by employing our proposed methodology, ARD value is 0.22 on day 1 , which further reduces on day 2 to 0.05 . Note that, ARD (Average Resource Deficit) values are lowest for the proposed methodology, as compared to those for the baselines, for both the days, as shown in Tables 24 and 25.

Figure 2 shows a visualization of the gradual decrease in ARD values at the end of Day 1 and Day 2 for the baseline methodologies and the proposed framework. From Fig. 2, it is evident that the proposed framework outperforms other baseline methodologies in terms of diminishing ARD values. It is also evident that the rate of decrease of ARD at the end of Day 1 and Day 2 is substantially higher for the proposed framework, than for the baselines.

\subsubsection{Fairness of resource allocation}

Apart from quickly bringing down resource-deficits, there is another important requirement for post-disaster resource allocation-the allocation of resources should be fair among various locations. Audits of resource allocation are often carried out after major disaster events, to ascertain if resources were fairly distributed among various disasteraffected locations. For instance, Paul et al. (2017) assessed whether emergency aid was uniformly distributed in different locations after the 2015 Nepal earthquake. Fairness issues have also been debated in the context of allocation of medical resources during the COVID-19 pandemic (Emanuel et al. 2020). Thus, while comparing different resource allocation models, it is important to consider how fair the allocation of resources is.

We now study how fair the three resource allocation models are in distributing resources among the different needlocations. To this end, we compare the resource allocation matrices of the three models for day 1 -Table 12 for the Simple Baseline, Table 16 for the Baseline based on location
Table 24 Comparing the three resource allocation models, at the end of day 1 -showing the $\operatorname{SRD}(t=1)$ value at each need-location (P1, $\mathrm{P} 2, \mathrm{P} 3, \mathrm{P} 4)$, and the average across need-locations $(A R D(t=1))$

\begin{tabular}{|c|c|c|c|c|c|}
\hline & $\mathrm{SRD}(\mathrm{P} 1)$ & $\mathrm{SRD}(\mathrm{P} 2)$ & SRD(P3) & $\mathrm{SRD}(\mathrm{P} 4)$ & ARD \\
\hline Simple Baseline & 0.25 & 0.50 & 0.00 & 1.00 & 0.44 \\
\hline $\begin{array}{l}\text { Baseline based on } \\
\text { location proxim- } \\
\text { ity }\end{array}$ & 0.83 & 1.00 & 0.00 & 0.20 & 0.51 \\
\hline $\begin{array}{l}\text { Proposed method- } \\
\text { ology }\end{array}$ & 0.33 & 0.00 & 0.53 & 0.00 & 0.22 \\
\hline
\end{tabular}

Table 25 Comparing the three resource allocation models, at the end of day 2-showing the $\operatorname{SRD}(t=2)$ value at each need-location (P1, $\mathrm{P} 2, \mathrm{P} 3, \mathrm{P} 4)$, and the average across need-locations $(A R D(t=2))$

\begin{tabular}{llllll}
\hline & SRD(P1) & SRD(P2) & SRD(P3) & SRD(P4) & ARD \\
\hline $\begin{array}{l}\text { Simple Baseline } \\
\text { Baseline based on } \\
\text { location proxim- }\end{array}$ & 0.00 & 0.12 & 1.00 & 0.00 & 0.28 \\
$\quad$ ity & 0.50 & 0.00 & 0.00 & 0.22 \\
$\begin{array}{l}\text { Proposed method- } \\
\text { ology }\end{array}$ & 0.00 & 0.21 & 0.00 & 0.00 & 0.05 \\
\hline
\end{tabular}

proximity, and Table 20 for the proposed method. Our proposed model enables the distribution of resources in a fair way among the different need-locations. For instance, on day $1, \mathrm{R} 1$ resource was needed in all four need-locations P1, P2, P3, P4 (see Table 7). The proposed model has allocated at least some amount of R1 to all the four need-locations (see Table 20). However, the Simple baseline methodology allocated R1 to P1, P2, P3 locations, but no allocation was made to P4 (Table 12). Thus, location P4 suffers from starvation problem. Likewise, while using the baseline scheme based on location proximity, allocation has been suggested for P1, P3, P4 and no allocation is suggested for P2 (Table 16), and thus $\mathrm{P} 2$ suffers from starvation.

Thus, it is evident that our proposed model can lead to resource allocation that is more fair. Since the baseline approaches consider each resource request in isolation, they may result in a situation where resources are allocated to only a subset of need-locations and other need-locations face starvation. In contrast, our proposed model considers the resource requests from all need-locations together, as a temporal sequence, and attempts to distribute the resources more evenly across all need-locations. Thus, it is much less likely that some particular need-location faces starvation of resources.

\subsubsection{Cost of achieving fairness in allocation}

It is well known that a methodology that strives to achieve fairness has to pay some associated 'cost'. For instance, a 
Average Resource Deficit Values Across the Need-locations at the end of DAY1 and DAY2 for Different Methodologies

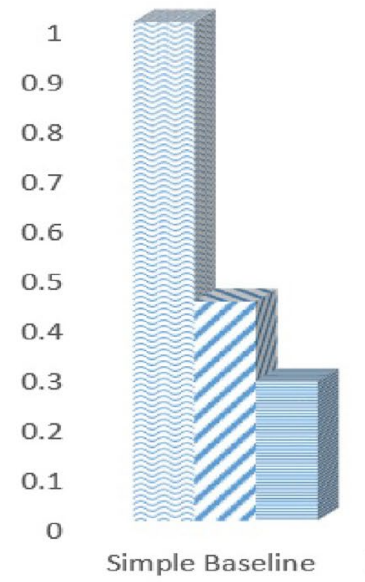

Simple Baseline

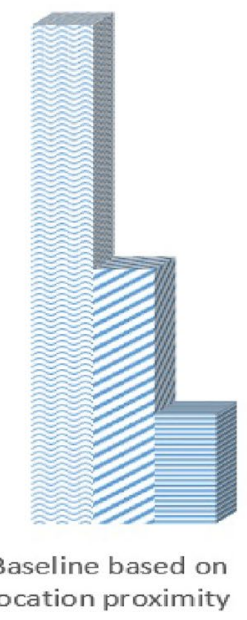

Baseline based on
location proximity

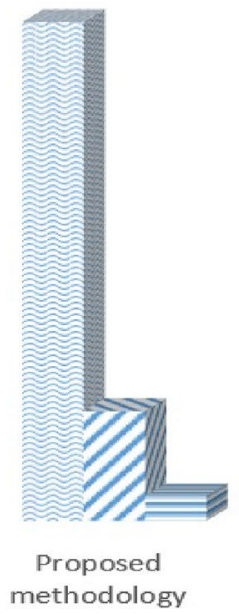

$\approx$ Initial

After DAY1

$\equiv$ After DAY2

Fig. 2 Average Resource Deficit values (averaged across all need-locations) at the end of Day 1 and Day 2. ARD is assumed to start at 1.0 for all cases (before deployment has started)

classifier that attempts to classify fairly often has to pay the cost of slightly reduced accuracy, compared to a classifier that optimizes only accuracy (Zafar et al. 2017). We now analyse the cost of achieving fairness in resource allocation, in terms of the time needed to deploy resources.

To this end, we define a new metric, called the Average Deployment Time (ADT) per unit of resource for a resource allocation model. This metric measures the average number of time units needed to deploy one unit of a resource following a specific methodology, averaged across all resources and over all days that are considered. For a particular resource deployment model, the metric considers the total time needed to deploy all the resources to all the need-locations from all warehouses. Then, ADT is computed by dividing the total time by the total number of unit of resources deployed. Thus, ADT for a particular model can be computed as

$\mathrm{ADT}=\frac{\sum_{t=1}^{N} \sum_{r=1}^{R} \sum_{p=1}^{P} \sum_{q=1}^{Q} t_{r p q} * y_{t r p q}}{\sum_{t=1}^{N} \sum_{r=1}^{R} \sum_{p=1}^{P} \sum_{q=1}^{Q} y_{t r p q}}$

where the symbols are as described in Table 4 and $N$ is the total number of days over which resource allocation takes place ( $N=2$ for our working example).

We compute ADT for the two baseline and the proposed models. For this computation, we utilize the deployment time matrix of the working example (Table 11) and the deployment matrices of the three methodologies. The computed ADT values are reported in Table 26.

From Table 26, it is evident that the Simple Baseline performs the best in terms of having low Average

Deployment Time per unit resource. However, our proposed methodology performs reasonably well in terms of ADT, and the deployment time is only slightly higher than that of the Simple Baseline. Thus, we can conclude that our proposed methodology achieves significant fairness with the reasonable cost of slightly increased deployment time of resources.

\section{Conclusion}

In this paper, we propose a model for optimized resource allocation in a post-disaster scenario, utilizing information posted on social media (specifically, tweets).

To the best of our knowledge, this is the first attempt to develop an optimized resource allocation framework utilizing social media data, which enables our framework to be used in real time. Specifically, we considered the information about resource-needs and resource-availabilities to be extracted from microblogging sites (which are possibly the most popular sources of real-time crowdsourced information today), and the model is adaptable to the imprecise information obtained from such sites. The evaluation using a working example derived from tweets posted during a real-life

Table 26 Measuring the cost of achieving fairness in resource allocation-Average Deployment Time per unit of resource, for the three methodologies

\begin{tabular}{lc}
\hline Method & ADT \\
\hline Simple Baseline & 29.62 \\
Baseline based on loca- & 33.27 \\
tion Proximity & \\
Proposed methodology & 30.82
\end{tabular}


disaster event (described in Sect. 5) shows that the proposed model outperforms the baselines in terms of bringing down resource-deficits (SRD values) at various need-locations. It is also evident that the rate of decrease in resource-deficits across need-locations (ARD values) is much higher for the proposed framework, than for the baseline methodologies. Additionally, the proposed model enables resource allocation that is efficient as well as more likely to be fair (i.e. resources are more uniformly distributed across all needlocations) than what baseline methods achieve.

There are several potential ways to improve the model in future. We used microblogging sites as the source of information since they provide real-time information, which is critical during a disaster. However, there are often concerns about false/exaggerated information on social media. Checking the correctness of resource-needs/resource-availabilites is out of the scope of the present work, but has to be included in order to deploy the model in practice. For instance, resource-needs and resource-availabilities can be input to the model only after proper verification. Also, though we have shown that the proposed model usually leads to more fair allocation than the baselines, the model can be improved in future by including provable guarantees of fairness in the allocation. It can also be noted that such a resource allocation model should be used in supervision of domain experts who, for instance, can decide the priority of various resource-needs by taking various local factors into account.

Acknowledgements The authors thank the anonymous reviewers for their valuable comments which helped to improve the paper.

\section{References}

Alqhtani SM, Luo S, Regan B (2018) A multiple kernel learning based fusion for earthquake detection from multimedia twitter data. Multimedia Tools Appl 77(10):12519-12532

Barnwal D, Ghelani S, Krishna R, Basu M, Ghosh S (2019) Identifying fact-checkable microblogs during disasters: a classificationranking approach. In: Proceedings of the 20th international conference on distributed computing and networking, ICDCN '19, pp 389-392

Basu M, Ghosh K, Das S, Dey R, Bandyopadhyay S, Ghosh S (2017a) Identifying post-disaster resource needs and availabilities from microblogs. In: Proceedings of ASONAM

Basu M, Ghosh S, Jana A, Bandyopadhyay S, Singh R (2017b) Resource mapping during a natural disaster: a case study on the 2015 Nepal earthquake. Int J Disaster Risk Reduct 24:24-31

Basu M, Shandilya A, Ghosh K, Ghosh S (2018a) Automatic matching of resource needs and availabilities in microblogs for post-disaster relief. In: Companion proceedings of the the web conference 2018 , international world wide web conferences steering committee, pp 25-26

Basu S, Roy S, Bandyopadhyay S, Bit SD (2018b) A utility driven post disaster emergency resource allocation system using dtn. IEEE Trans Syst Man Cybern Syst 50(7):2338-2350. https://doi.org/ 10.1109/TSMC.2018.2813008
Basu M, Shandilya A, Khosla P, Ghosh K, Ghosh S (2019) Extracting resource needs and availabilities from microblogs for aiding post-disaster relief operations. IEEE Trans Comput Soc Syst 6(3):604-618

Basu M, Bit SD, Ghosh S (2021) Utilising social media for post-disaster resource allocation. In: 8th ACM IKDD CODS and 26th COMAD, p 440

Bhavaraju SKT, Beyney C, Nicholson C (2019) Quantitative analysis of social media sensitivity to natural disasters. Int J Disaster Risk Reduct 39:101251. https://doi.org/10.1016/j.ijdrr.2019.101251

Bhoi A, Pujari SP, Balabantaray RC (2020) A deep learning-based social media text analysis framework for disaster resource management. Soc Netw Anal Min 10(1):1-14

Daneshfar F, Kabudian SJ (2020) Speech emotion recognition using discriminative dimension reduction by employing a modified quantum-behaved particle swarm optimization algorithm. Multimedia Tools Appl 79(1):1261-1289

Disaster-uttarakhand (2013) Resource allocation for Uttarakhand disaster 2013, Disaster Mitigation Management Centre. http://dmmc. uk.gov.in/pages/view/72

Dutt R, Basu M, Ghosh K, Ghosh S (2019) Utilizing microblogs for assisting post-disaster relief operations via matching resource needs and availabilities. Inf Process Manag 56(5):1680-1697

Emanuel EJ, Persad G, Upshur R, Thome B, Parker M, Glickman A, Zhang C, Boyle C, Smith M, Phillips JP (2020) Fair allocation of scarce medical resources in the time of covid-19. N Engl J Med 382:2049-2055

Gershuny J (2009) Activities, durations and the empirical estimation of utility. Sociology Working Papers 7

Hiltz SR, Hughes AL, Imran M, Plotnick L, Power R, Turoff M (2020) Exploring the usefulness and feasibility of software requirements for social media use in emergency management. Int J Disaster Risk Reduct 42:101367. https://doi.org/10.1016/j.ijdrr.2019. 101367

Imran M, Castillo C, Diaz F, Vieweg S (2015) Processing social media messages in mass emergency: a survey. ACM Comput Surv 47(4): $1-38$

Khosla P, Basu M, Ghosh K, Ghosh S (2017) Microblog retrieval for post-disaster relief: applying and comparing neural IR models. In: SIGIR 2017 workshop on neural information retrieval (Neu-IR'17)

Kondaveti R, Ganz A (2009) Decision support system for resource allocation in disaster management. In: Proceedings of international conference of the IEEE engineering in medicine and biology society, pp 3425-3428

Kumar A, Singh JP (2019) Location reference identification from tweets during emergencies: a deep learning approach. Int J Disaster Risk Reduct 33:365-375

Kumar A, Sangwan SR, Nayyar A (2019) Rumour veracity detection on twitter using particle swarm optimized shallow classifiers. Multimedia Tools Appl 78(17):24083-24101

Kumar A, Singh JP, Dwivedi YK, Rana NP (2020) A deep multimodal neural network for informative twitter content classification during emergencies. Ann Oper Res. https://doi.org/10.1007/ s10479-020-03514-x

Kuo WH, Liao W (2007) Utility-based resource allocation in wireless networks. IEEE Trans Wirel Commun 6(10):3600-3606

Li T, Xie N, Zeng C, Zhou W, Zheng L, Jiang Y, Yang Y, Ha HY, Xue W, Huang Y, Chen SC, Navlakha J, Iyengar SS (2017) Data-driven techniques in disaster information management. ACM Comput Surv 50(1): 1-45

Madichetty S, Sridevi M (2020a) Classifying informative and noninformative tweets from the twitter by adapting image features during disaster. Multimed Tools Appl 79(39):28901-28923 
Madichetty S, Sridevi M (2020) Identification of medical resource tweets using majority voting-based ensemble during disaster. Social Netw Anal Min 10(1):1-18

McConnell C, Brue S, Flynn S (2014) Law of diminishing marginal utility. McGraw-Hill

Paul B, Acharya B, Ghimire K (2017) Effectiveness of earthquakes relief efforts in Nepal: opinions of the survivors. Nat Hazards 85:1169-1188

Pohl D, Bouchachia A, Hellwagner H (2015) Social media for crisis management: clustering approaches for sub-event detection. Multimedia Tools Appl 74(11):3901-3932

Pourebrahim N, Sultana S, Edwards J, Gochanour A, Mohanty S (2019) Understanding communication dynamics on twitter during natural disasters: a case study of hurricane sandy. Int J Disaster Risk Reduct 37:101176. https://doi.org/10.1016/j.ijdrr.2019.101176

Purohit H, Castillo C, Diaz F, Sheth A, Meier P (2014) Emergencyrelief coordination on social media: automatically matching resource requests and offers. First Monday 19(1). https://doi.org/ 10.5210/fm.v19i1.4848

Purohit H, Castillo C, Pandey R (2020) Ranking and grouping social media requests for emergency services using serviceability model. Social Netw Anal Min 10(1):1-17

Radianti Z, Granmo OC, Sarshar P, Goodwin M, Dugdale J, Gonzalez JJ (2015) A spatio-temporal probabilistic model of hazard- and crowd dynamics for evacuation planning in disasters. Appl Intell 42(2):3-23

Rudra K, Ghosh S, Goyal P, Ganguly N, Ghosh S (2015) Extracting situational information from microblogs during disaster events: a classification-summarization approach. In: Proceedings of ACM CIKM
Schempp T, Zhang H, Schmidt A, Hong M, Akerkar R (2019) A framework to integrate social media and authoritative data for disaster relief detection and distribution optimization. Int J Disaster Risk Reduct 39:101143. https://doi.org/10.1016/j.ijdrr.2019.101143

Singh JP, Dwivedi YK, Rana NP, Kumar A, Kapoor KK (2019) Event classification and location prediction from tweets during disasters. Ann Oper Res 283(1):737-757

Utility-clams (2016) Utility of Clams. http://sandovalhernandezj.people.cofc.edu/index_files/ch10.pdf

Utility-marginal (2016) Total Utility vs. Marginal Utility. http://www. yourarticlelibrary.com/managerial-825economics/ total-utility-vsmarginal-utility-explained-with-diagram/28383/

Varga I et al (2013) Aid is out there: looking for help from tweets during a large scale disaster. In: Proceedings of ACL

Xu Z, Liu Y, Xuan J, Chen H, Mei L (2017) Crowdsourcing based social media data analysis of urban emergency events. Multimedia Tools Appl 76(9):11567-11584

Yadav M, Rahman Z (2016) The social role of social media: the case of Chennai rains-2015. Social Netw Anal Min 6(1):1-12

Zafar MB, Valera I, Rodriguez MG, Gummadi KP (2017) Fairness constraints: mechanisms for fair classification. In: Proceedings of international conference on artificial intelligence and statistics (AISTATS), pp 962-970

Publisher's Note Springer Nature remains neutral with regard to jurisdictional claims in published maps and institutional affiliations. 